Algebraic 83 Geometric $\mathcal{T}$ opology

Volume 5 (2005) 443-462

Published: 26 May 2005

ATG

\title{
Signed ordered knotlike quandle presentations
}

\author{
SAM NELSON
}

\begin{abstract}
We define enhanced presentations of quandles via generators and relations with additional information comprising signed operations and an order structure on the set of generators. Such a presentation determines a virtual link diagram up to virtual moves. We list formal Reidemeister moves in which Tietze moves on the presented quandle are accompanied by corresponding changes to the order structure. Omitting the order structure corresponds to replacing virtual isotopy by welded isotopy.
\end{abstract}

AMS Classification 57M25, 57M27; 27M05, 20F05

Keywords Quandles, virtual knots, presentations, Reidemeister moves, welded isotopy

Dedicated to the memory of my mother, Linda M Nelson, who was killed in an automobile accident while this paper was in preparation.

\section{Introduction}

The knot quandle was defined in [10] and has been studied in a number of subsequent works. It is well known that the knot quandle is a classifying invariant for classical knots, in the sense that the quandle contains the same information as the fundamental group system and hence determines knots and links up to (not necessarily orientation-preserving) homeomorphism of pairs. Whether the quandle alone is a complete invariant of knot type, however, depends on the meaning of "equivalence" - in particular, if "equivalent" means "ambient isotopic", then the quandle alone does not classify knots. Indeed, there are examples of pairs of classical (and virtual) knots with isomorphic knot quandles which are not ambient (or virtually) isotopic, such as the left and right hand trefoil knots. This shows that a quandle isomorphism need not translate to a Reidemeister move sequence. Moreover, Reidemeister moves on a knot diagram correspond to sequences of Tietze moves on the presented quandle, but the converse is not generally true. We wish, then, to characterize which Tietze moves on quandle presentations do correspond to Reidemeister moves 
and hence preserve ambient (or virtual) isotopy class. We will assume that all knots are endowed with a choice of orientation, ie, we will concern ourselves only with oriented knots and links.

Examples of nontrivial virtual knots with trivial knot quandle are given in [3. Virtual knots are equivalence classes, under the equivalence relation generated by the three Reidemeister moves, of 4-valent graphs (both planar and nonplanar) with vertices interpreted as crossings; classical knot theory may be regarded as the special case of virtual knot theory in which we restrict our attention to planar graphs. Virtual knots have two associated quandles, an upper quandle defined in the usual combinatorial way (described in section 3) while ignoring any virtual crossings, and a lower quandle, defined as the upper quandle of the knot diagram obtained by "flipping over" the original knot diagram by taking a mirror image and switching all under/overcrossings. If the knot is classical, the "flipping over" operation is an ambient isotopy, and the resulting upper and lower quandles are isomorphic, though the quandle presentations defined by the knot diagrams may bear little obvious resemblance. If the knot is not classical, however, the upper and lower quandles are typically distinct; see 9].

One approach to the problem of finding a complete algebraic invariant for virtual knots involves combining the upper and lower quandles into a single algebraic structure, namely the biquandle (defined in [7] and applied to virtual knots in [4). In this paper we take a different approach, defining additional structure on the usual presentation of the upper quandle which permits reconstruction of a virtual knot diagram from the enhanced presentation.

In section 2, we recall presentations of quandles by generators and relations, noting that every finite quandle has a presentation resembling that determined by a knot or link diagram. In section 3 we recall some facts about virtual knots and the definition of the knot quandle, then present our primary definition, signed ordered knotlike quandle presentations. In section 4, we examine how the Reidemeister moves affect the order structure, defining formal Reidemeister moves on SOKQ presentations. In section 5, we show how to apply our results to welded isotopy classes (also known as weak virtual isotopy classes) and in section 6 extend to cover the framed case.

\section{Quandle presentations}

A quandle is a set $Q$ with a non-associative binary operation $\triangleright: Q \times Q \rightarrow Q$ satisfying 
(qi) for every $a \in Q$, we have $a \triangleright a=a$,

(qii) for every $a, b \in Q$ there exists a unique $c \in Q$ with $a=c \triangleright b$, and

(qiii) for every $a, b, c \in Q$, we have $(a \triangleright b) \triangleright c=(a \triangleright c) \triangleright(b \triangleright c)$.

As expected, a homomorphism of quandles is map $f: Q \rightarrow Q^{\prime}$ such that

$$
f(x \triangleright y)=f(x) \triangleright f(y),
$$

and a bijective homomorphism of quandles is an isomorphism of quandles. The quandle operation is asymmetrical; it is an action of the set $Q$ on itself. Several authors have written on quandles, and a number of different notational styles are in common use: [6] uses exponential notation, where $a \triangleright b$ is denoted $a^{b}$, while 1] uses an asterisk $*$ in place of the triangle $\triangleright$. Moreover, some authors put the action on the right, while others put it on the left.

For the purpose of defining quandles via presentations, it is convenient to follow [10] using the triangle notation with the action on the right, so that $x \triangleright y$ means "the result of the action of $y$ on $x$." The existence and uniqueness requirements of axiom (qii) imply that each quandle comes with a second operation $\triangleleft$ satisfying $(x \triangleleft y) \triangleright y=x$. Specifically, axiom (qii) is equivalent to the statement that for all $y \in X$, the map $f_{y}(x)=x \triangleright y$ is a bijection. We may then denote $f_{y}^{-1}(x)=x \triangleleft y$, and we have $(x \triangleright y) \triangleleft y=f_{y}^{-1}\left(f_{y}(x)\right)=x$ and $(x \triangleleft y) \triangleright y=f_{y}\left(f_{y}^{-1}(x)\right)=x$. This operation $\triangleleft: Q \times Q \rightarrow Q$ itself defines a quandle structure on $Q$, called the dual of $Q$. A quandle is self-dual if it is isomorphic to its dual, that is, if there is a bijection $f: Q \rightarrow Q$ with $f(x \triangleright y)=f(x) \triangleleft f(y)$. The dual operation $x \triangleleft y$ is also denoted $x^{\bar{y}}, x \bar{*} y$, or $x \triangleright^{-1} y$.

We will use the symbol $\diamond \in\{\triangleright, \triangleleft\}$ as a generic quandle operation, so the notation $x \diamond y$ can mean either $x \triangleright y$ or $x \triangleleft y$. When we have already used $\diamond$ in a formula, we will use $\bar{\diamond}$ to specify the opposite operation, and when we need to specify several possibly different quandle operations, we will use subscripts: $\diamond_{1}, \diamond_{2}$ etc.

Although the quandle operations are not associative, we can write any element of the quandle in the form

$$
\left(\ldots\left(\left(\left(x_{1} \diamond_{1} x_{2}\right) \diamond_{2} x_{3}\right) \diamond_{3} \ldots\right) \diamond_{n-1} x_{n}\right),
$$

since $\quad x \diamond_{1}\left(y \diamond_{2} z\right)=\left(\left(x \bar{\diamond}_{2} z\right) \diamond_{2} z\right) \diamond_{1}\left(y \diamond_{2} z\right)=\left(\left(\left(x \bar{\diamond}_{2} z\right) \diamond_{1} y\right) \diamond_{2} z\right)$.

This form is called fully left assocated and we shall often assume, without comment, that words are presented in this form. The length of such a word is the number of operations ( $\triangleright$ or $\triangleleft$ ) which occur. 
We refer to [6] for basic results about presentations of quandles, free quandles and the Tietze theorem. [6] is written in terms of the more general concept of racks (axiom (qi) is omitted) but the treatment specialises to quandles in the obvious way. A quandle presentation can contain both primary and secondary (or operator) generators and relations. Here we shall only be concerned with primary generators and relations. Such a presentation has the form $\langle X \mid R\rangle$, where $X=\left\{x_{1}, \ldots, x_{n}\right\}$ is the generating set and $R=\left\{r_{i} \sim r_{i}^{\prime}, i=1 \ldots m\right\}$ is the set of relations. Here each $r_{i}$ and $r_{i}^{\prime}$ is a word in $x_{1}, \ldots, x_{n}$.

Definition 2.1 A relation of the form $x \sim y \diamond z$ where $x, y$, and $z$ are generators is a short relation. In a relation of this form, the generator $x$ is the output operand, $y$ is the input operand, and $z$ is the operator.

Proposition 2.1 Every finitely presented quandle $\langle X \mid R\rangle$ has a presentation such that

- every relation is short,

- at most one of $\left\{x \sim y \diamond z, x^{\prime} \sim y \diamond z\right\}$ appears in $R$,

- at most one of $\left\{x \sim y \diamond z, x \sim y^{\prime} \diamond z\right\}$ appears in $R$,

- at most one of $\{x \sim y \triangleright z, y \sim x \triangleleft z\}$ appears in $R$,

- at most one of $\{x \sim y \triangleright y, x \sim y \triangleleft y\}$ appears in $R$.

Proof Every relation has the form $w_{1} \sim w_{2}$ where $w_{i}$ are words in the generators. We may assume $w_{1}, w_{2}$ are not both generators, since if they are, we can delete one and replace every occurrence of the deleted generator with the equivalent generator without changing the quandle. Each word $w_{i}$ can be written in fully left associated form, so we may further assume every relation has the form

$$
x_{1} \diamond_{1} x_{2} \diamond_{2} \ldots \diamond_{n-1} x_{n} \sim y_{1} \diamond_{n+1} y_{2} \diamond_{n+2} \ldots \diamond_{n+m-1} y_{m}
$$

If we already have a relation $z \sim x_{1} \diamond_{1} x_{2}$, we can replace $x_{1} \diamond_{1} x_{2}$ with $z$, shortening the left hand side of the relation; if not, we can introduce a new generator $z$ with defining relation $z \sim x_{1} \diamond_{1} x_{2}$ and do the replacement. Repeating the procedure, we can reduce the left side (say) to a single generator and the right side to a word $u \diamond v$ of length one, so that all relations have the short form $x \sim y \diamond z$.

If both $x \sim y \diamond z$ and $x^{\prime} \sim y \diamond z$ are in $R$, then $x \sim x^{\prime}$ and replacing all instances of $x^{\prime}$ with $x$ and deleting the relation $x^{\prime} \sim y \diamond z$ and the generator $x^{\prime}$ yields 
an isomorphic quandle. Similarly, since $x \sim y \diamond z$ is equivalent to $y \sim x \bar{\diamond} z$, the third claim reduces to the second.

By the quandle laws $x \sim y \triangleright z$ implies $x \triangleleft z \sim(y \triangleright z) \triangleleft z$ which implies $x \triangleleft z \sim y$. Hence the relations $x \sim y \triangleright z$ and $y \sim x \triangleleft z$ are equivalent, so we can choose one and delete the other without changing the presented quandle. Similarly, $x \sim y \triangleright y$ is equivalent to $x \sim y \triangleleft y$.

Remark It is also true that $x \sim y \triangleright y$ is equivalent to $x \sim y$ by (qi) and therefore relations of the form $x \sim y \triangleright y$ can be removed from the presentation. However it is convenient to keep these redundant relations as they occur naturally in presentations coming from diagrams. They are also necessary for the analogous framed case, where racks are used rather than quandles and axiom (qi) is not assumed to hold (see section [6). Note also that the relations $x \sim y \diamond z$ and $x \sim y \diamond z^{\prime}$ are independent, in general.

Definition 2.2 A quandle presentation $Q=\langle X \mid R\rangle$ of the type described in the preceding proposition is in short from.

A virtual knot or link diagram determines a quandle presentation in short form, as we shall see in the next section. In particular, the quandle presentations determined by the diagrams before and after a Reidemeister move are both in short form, so a sequence of Tietze moves on a knot quandle must determine a sequence of short-form presentations in order to correspond to a sequence of Reidemeister moves.

Since every quandle has a short form presentation, it is natural to ask when a virtual knot diagram can be reconstructed from a short form quandle presentation and to what degree the isomorphism class of the quandle determines the resulting virtual knot. We will see that a short form quandle presentation meeting certain sufficient conditions, together with some additional structure, determines a virtual link diagram.

\section{Knotlike presentations and link diagrams}

Interest in quandles is motivated primarily by their utility in defining invariants of knots and links, disjoint unions of embedded circles in $S^{3}$ or another 3 -manifold. A knot or link with a choice of orientation is an oriented knot or link. Oriented knots and links in $S^{3}$ may be studied combinatorially as equivalence classes of link diagrams, 4 -valent graphs with two edges oriented 
in and two oriented out at every vertex, with vertices interpreted as crossings and enhanced with crossing information, under the equivalence relation generated by the three familiar Reidemeister moves. If the underlying graph of a link diagram is a plane graph, ie, a planar graph actually embedded in the plane, the link diagram is classical; otherwise, it is virtual. Equivalence classes of oriented link diagrams under the Reidemeister moves correspond to ambient isotopy classes of embedded disjoint oriented circles in $S^{3}$ if the link diagrams are classical and stable equivalence classes of embedded disjoint oriented circles in thickened surfaces $S \times[0,1]$ if the diagrams are virtual; see [2, 8, 12].

If the graph is non-planar, any additional crossings which must be introduced in order to draw the graph in the plane are virtual crossings, self-intersections which are circled to distinguish them from real crossings. Any arc containing only virtual crossings may be replaced by any other arc with the same endpoints and only virtual crossings in a detour move to obtain an equivalent virtual link diagram. The equivalence relation on link diagrams generated by the three Reidemeister moves and the detour move is called virtual isotopy. If, in addition, a strand with two classical overcrossings is permitted to move past a virtual crossing, figure 1 we have the forbidden move $F_{h}$, and the equivalence relation generated by virtual isotopy and $F_{h}$ is known as welded isotopy or weak virtual isotopy; see [5, 11. Welded links (ie welded isotopy classes of virtual link diagrams) are important because of their close connection with the braid-permutation group [5].

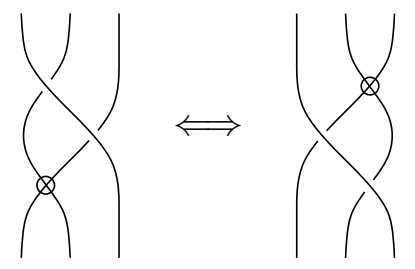

Figure 1: The "forbidden move" $F_{h}$

An oriented link diagram is a link diagram in which the edges are oriented in a coherent manner; specifically, of the two edges comprising the overstrand according to the crossing information, one is oriented toward the vertex and the other away from the vertex, and similarly for the undercrossing strand. These arcs are then unions of edges meeting at overcrossings.

Crossings are given signs according to their local writhe number. A crossing is positive if the orientation on the plane determined by the orientations on the 
overarc followed by the underarc(s) gives the standard right-hand orientation on the plane; otherwise, the crossing is negative.

In [10] and 6], combinatorial rules are given for associating a quandle to an oriented link diagram, with a generator for every arc and a relation at each crossing. In [10], the link diagrams are unoriented, with the relation determined by the blackboard framing of the link diagram, while in [6] the orientation of the over-crossing strand (but the not the under-crossing strand) determines the relation. Specifically, if we look in the positive direction of the overstrand $y$, we obtain the relation $x \triangleright y \sim z$ where $x$ is the undercrossing edge on the right and $z$ is the undercrossing edge on the left. The quandle presented in this way is the knot quandle of the diagram; the fact that the knot quandle is an invariant of oriented knots and links is easily checked by comparing the presentations determined by the diagram before and after each of the Reidemeister moves, keeping the quandle axioms in mind.
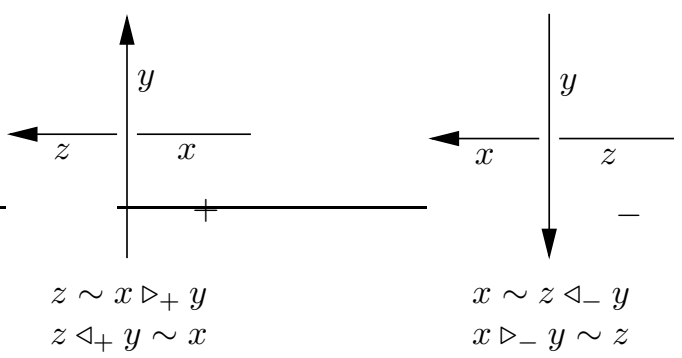

Figure 2: Crossing signs and quandle operations

The fact that $x \triangleright y \sim z$ is equivalent to $x \sim z \triangleleft y$ shows that this rule determines not one unique relation but a pair of equivalent relations. This poses a difficulty when we wish to attempt to reconstruct a link diagram from its presented quandle; a short relation $x \sim z \triangleright y$ determines everything about a crossing except the orientation of the undercrossing strand. We can avoid this difficulty by including the crossing sign as a subscript with each quandle operation.

It is then easy to see that this subscript convention yields information about which Tietze moves translate to Reidemeister moves and which do not. For instance, in every type II move, the two crossings have opposite signs, whereas if we rewrite a short form relation with the other quandle operation, both operations must have the same sign. This extra information is invisible to the quandle structure and thus defines an enhanced quandle presentation, called a signed quandle presentation. 
Not every combination of sign choice for each short form relation corresponds to a virtual link diagram; we must have exactly one inbound (that is, oriented toward the vertex) and one outbound (oriented away from the vertex) undercrossing arc at each crossing in order to have a virtual knot or link diagram. With our reconstruction rule, if the quandle operation is $\triangleright_{+}$the input operand is inbound and the output operand is outbound. Switching either the sign or the triangle switches which operand is inbound and which is outbound. Simultaneously switching all of the signs gives us the quandle of the reflection of the virtual link, while switching the signs of a proper subset of relations in a presentation corresponding to a diagram yields an incoherent signed quandle presentation - one which does not correspond to a virtual link diagram.

Indeed, since a choice of sign for one crossing determines which operand is inbound and which is outbound, such a choice also determines which operands are inbound and outbound in the other relations in which these generators appear are operands, and thus determines a sign for the crossings meeting the other ends of the undercrossing arcs. Likewise, each of these crossing signs determines the signs of the other crossings containing these operands, and so on. In all, there are $2^{N}$ possible coherent quandle presentations for a given set of short relations, where $N$ is the number of components with at least one undercrossing.

Using the rule that distinct generators are assigned to every arc in an oriented knot diagram, we notice that every arc is either a simple closed curve or has endpoints at crossings; hence in a knot quandle presentation, every generator is either an operator-only generator or appears as an inbound operand exactly once and as an outbound operand exactly once in the set of relations. Moreover, every relation determined by a crossing is in short form, and it is not hard to check that the remaining conditions in definition 2.1 are fulfilled.

Thus, the quandle presentation determined by a virtual link diagram is a short form quandle presentation. The converse is not true in general, since generators can appear more than twice as operands in a general short-form quandle presentation - for example,

$$
\langle x, y, z, w \mid x \triangleright y \sim z, y \triangleright z \sim x, z \triangleright x \sim y, x \triangleright w \sim z\rangle .
$$

Definition 3.1 A coherent short form signed quandle presentation is knotlike if every generator either appears in exactly one relation as an inbound operand and in exactly one relation as an outbound operand, or appears only as an operator. A quandle is knotlike if it has a knotlike presentation. 
It is clear that every virtual link diagram defines a knotlike quandle presentation. Furthermore is it easy to see that every knotlike quandle presentation determines a virtual link diagram; however a little investigation shows that in most cases there are several distinct virtual knot diagrams which all define the same signed quandle presentation, eg, the inequivalent virtual knots pictured in figures 3 and 4. In light of this, it is natural to ask to what degree a virtual link is determined by a knotlike quandle presentation.

We can begin constructing a virtual link diagram from a knotlike quandle presentation by interpreting each signed short form relation as specifying a crossing, according to the reconstruction rule. The condition that every generator either appears exactly one relation as an inbound operand and exactly one relation as an outbound operand or only in the operator position means that every arc either has a well-defined initial and terminal point or is a simple closed curve, and we can complete the diagram by joining initial and terminal points with the same label with an arc including all the overcrossings of the same label, making virtual crossings as necessary.

The virtual knot diagram so constructed is not unique if any generator appears more than once as an operator. Indeed, for every such generator, we must choose an order in which to go through the overcrossings along the arc, and different choices can result in non-isotopic virtual knot diagrams, all of which necessarily have the same quandle. Figure 3 depicts the two simplest inequivalent virtual knot diagrams constructed from the same quandle; the one on the left is Kauffman's virtualized trefoil, which is known to be non-trivial [11, while the diagram on the right is an unknot.

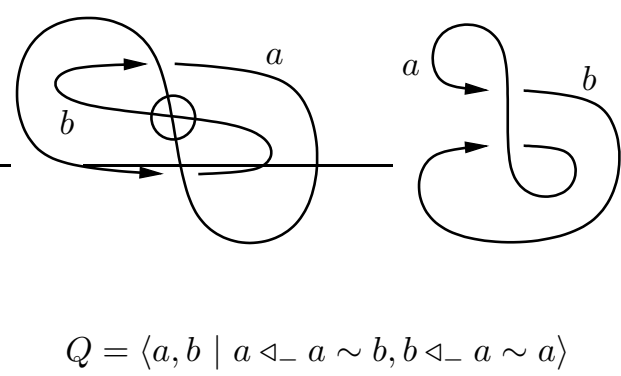

Figure 3: Example of non-isotopic virtual knots constructed from the same knotlike quandle presentation

Figure 4 shows another pair of known distinct virtual knots constructed from the same knotlike quandle presentation, differing only in the order information; 
the virtual knot on the left in one of the Kishino virtual knots, known to be non-trivial, while the one on the right again is an unknot.

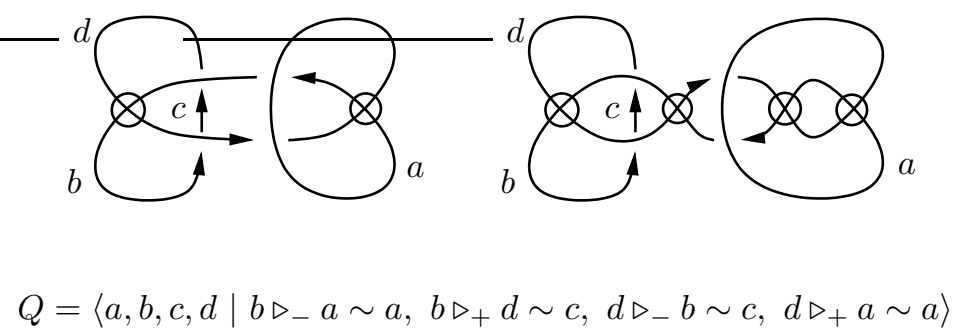

Figure 4: Another example of non-isotopic virtual knots constructed from the same knotlike quandle presentation

Figures 3 and 4 show that signed quandle presentations alone are not sufficient to distinguish all oriented virtual knots. In order to completely specify a virtual knot diagram, we must find a way of indicating an order for the overcrossings. In an oriented knot diagram, each arc has a well-defined direction which we may use to order the crossings; indeed, this is precisely what one does when describing a virtual knot diagram by means of a Gauss code. Moreover, since every crossing includes precisely one incoming undercrossing strand, there is a bijection between the set of generators and the set of crossings. Thus, the order of the inbound undercrossings along a given arc in a virtual link diagram defines a partial order on the set of generators, with two generators being comparable if and only if the terminal points of their arcs lie along the same overcrossing arc. Call the sets of generators whose terminal points lie along the the same arc arc-comparison classes.

In addition to ordering crossings along arcs, a virtual knot diagram also defines a cyclic order in which the arcs are encountered while moving along a component. If the diagram is a virtual link, this cyclic ordering of arcs is a cyclic partial order on the arc-comparison classes of generators; if the diagram is a virtual knot, we have a cyclic order on these ordered sets.

Definition 3.2 Let $K$ be a virtual knot diagram and $Q=\langle X \mid R\rangle$ the corresponding knotlike quandle presentation. Say that $a<b$ if the terminal points of the arcs labeled $a$ and $b$ occur in crossings along the same arc $z$ and if traveling along $z$ from its initial point to its terminal point we encounter the terminal point of $a$ before the terminal point of $b$. 
Let $C_{a}$ be the arc-comparison class of $a$ under $<$. Then we have a cyclic partial ordering $\prec$ on the set $\left\{C_{x} \mid x \in X\right\}$ defined by $C_{a} \prec C_{b}$ if the terminal point of $a$ meets the initial point of $b$ at a crossing. If the class $C_{a}$ consists of generators $x_{1}<x_{2}<\ldots<x_{n}$, we may write $C_{a}=\underline{x_{1} x_{2} \ldots x_{n}}$. If $C_{a}$ is empty, we may write $C_{a}=$

If $C_{a_{1}} \prec C_{a_{2}} \prec \ldots \prec C_{a_{n}} \prec C_{a_{1}}$, we may simply write $\left(C_{a_{1}} C_{a_{2}} \ldots C_{a_{n}}\right)$, and if $K$ has multiple components, we may specify the order information of $K$ with a comma-separated list of these cyclically ordered partial order classes.

Alternatively, we may specify the the order information implicitly by simply listing the relations in the order specified by the order information, with each relation's position determined by its inbound operand. The comparison class of a generator $a$ then includes all relations with operator $a$.

Since a generator $a$ is comparable to $b$ iff the terminal points of both corresponding arcs lie along the same overcrossing arc, $<$ is a partial order on the set of generators. Moreover, $<$ is clearly strict. If a generator $z$ is operator-only, its class $C_{z}$ will form its own class under $\prec$; in particular, the partial order $<$ on $C_{z}$ is actually a cyclic order on $C_{z}$ in this case, and only in this case - the arc-comparison class of any non-operator-only generator has a maximal element and minimal element, or is empty.

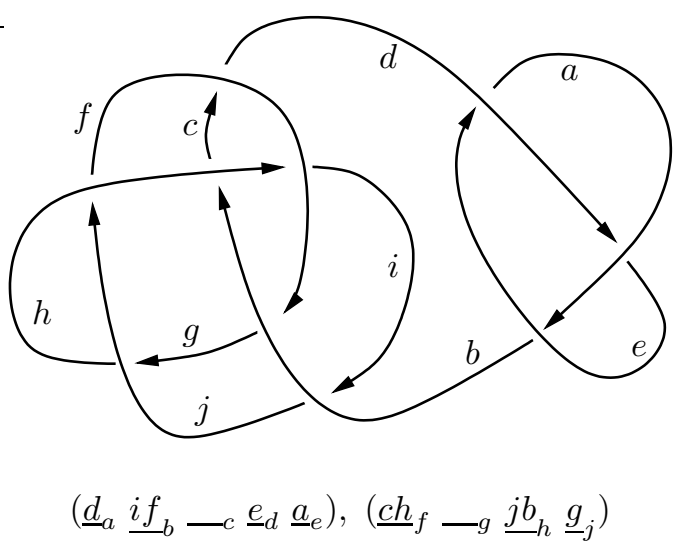

Figure 5: An example of order information from a link diagram

From a virtual knot digram we can read off this order information and include it with a knotlike quandle presentation to obtain a signed ordered knotlike quandle presentation or a $S O K Q$ presentation. Conversely, given a signed ordered 
knotlike quandle presentation, we can construct a virtual link diagram which is uniquely determined up to virtual moves.

\section{Formal Reidemeister moves}

In this section we note how Reidemeister moves on a virtual knot diagram change an ordered quandle presentation.

Reidemeister type I and II moves come in two varieties, crossing-introducing and crossing-removing. The crossing-introducing Reidemeister type I move breaks an arc into two and introduces a crossing, while the crossing-removing type I move removes a crossing and joins two arcs which previously met at an undercrossing into one. The crossing-introducing type II move introduces two crossings and breaks one arc into three, while the crossing-removing type II move removes two crossings and joins three arcs into one.

Let us use the convention that in a type I or II move, the original generator name stays with the terminal point of the arc. Then the crossing-introducing type I move

- breaks the arc-comparison class $C_{a}=x_{1} \ldots x_{n_{a}}$ into two classes, either $C_{a^{\prime}}={\underline{x_{1} \ldots x_{i} a^{\prime}}}_{a^{\prime}}$ and $C_{a}=\underline{x i+1}_{a^{\prime}} \ldots \bar{x}_{n_{a}}$ or $C_{a^{\prime}}=\underline{x_{1} \ldots x_{i}} a_{a^{\prime}}$ and $C_{a}=$ $\underline{a^{\prime} x_{i+1} \ldots x_{n}} a$

- introduces a new generator $a^{\prime}$ and relation $a \sim a^{\prime} \triangleright_{+} a^{\prime}$ or $a \sim a^{\prime} \triangleleft_{-} a^{\prime}$ in the first case or $a^{\prime} \sim a \triangleright_{-} a$ or $a^{\prime} \sim a \triangleleft_{+} a$ in the second case, and

- replaces the operator $a$ with $a^{\prime}$ in the relations with inbound operand $x_{1}, \cdots x_{i}$ and replaces the outbound operand $a$ with $a^{\prime}$ in its relation.

Conversely, a crossing-removing type I move is only available in an ordered quandle presentation if the arc-comparison classes $C_{a}^{\prime} \prec C_{a}^{\prime}$ are adjacent in the cyclic order, with $C_{a^{\prime}}$ ending in $a^{\prime}$ if the relation is $a \sim a^{\prime} \triangleright_{+} a^{\prime}$ or $a \sim a^{\prime} \triangleleft_{-} a^{\prime}$, or $C_{a}$ starting with $a^{\prime}$ if the relation is $a^{\prime} \sim a \triangleright_{-} a$ or $a^{\prime} \sim a \triangleleft_{+} a$. In this case, we can delete the generator $a^{\prime}$ and the relation with inbound operand $a^{\prime}$, replace every instance of $a^{\prime}$ with $a$, and join $C_{a^{\prime}}$ with $C_{a}$ in order, minus the $a^{\prime}$, to form the new $C_{a}$ and thus obtain the new signed ordered quandle presentation. See figure 6 for an illustration.

Either of these operations on an ordered quandle presentation will be called formal Reidemeister move I. Note that in terms of Tietze moves, we've simply introduced a new generator $a^{\prime}$ and defining relation $a^{\prime} \sim a$, replaced some instances of $a$ with $a^{\prime}$ and made the presentation knotlike by replacing $a \sim a^{\prime}$ 
with an equivalent relation. Absent the order information, we can replace any, all, or none of the operator occurrences of $a$ with $a^{\prime}$ to obtain an equivalent quandle, while most such moves will not correspond to Reidemeister moves or move sequences, though many may be realizable as welded isotopy sequences.

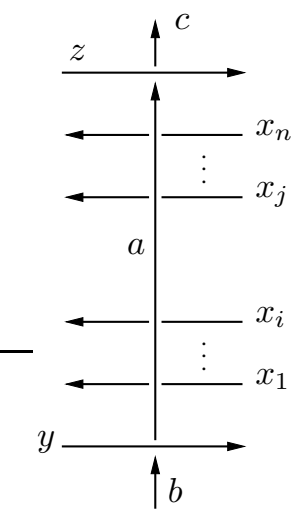

$b \triangleright_{+} y \sim a, a \triangleright_{+} z \sim c$

$\underline{x_{1}, \ldots, x_{n}} a$

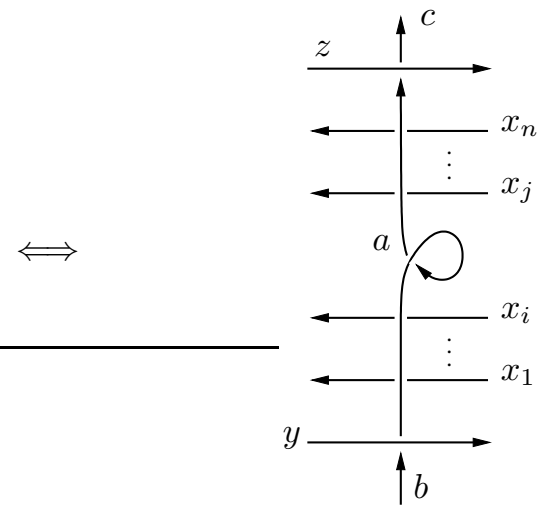

$b \triangleright_{+} y \sim a^{\prime}, a^{\prime} \triangleright_{+} a^{\prime} \sim a, a \triangleright_{+} z \sim c$

$\underline{x_{1}, \ldots, x_{i} a^{\prime}} a^{\prime} \underline{x_{i+1}, \ldots, x_{n}} a$

operator $a$ replaced with $a^{\prime}$ in relations involving inbound operand $x_{1} \ldots x_{i}$

Figure 6: Formal Reidemeister move I example

A crossing-introducing Reidemeister II move breaks an arc-comparison class into three classes while introducing two generators, two relations, and replacing some instances of one generator with a new one. Specifically,

- the arc-comparison class $C_{a}=x_{1} \ldots x_{n_{a}}$ is replaced by $C_{a^{\prime \prime}} C_{a^{\prime}} C_{a}=$ $\underline{x}_{1} \ldots x_{i} a^{\prime \prime}-a^{\prime} x_{i+1} \ldots x_{n}$ and the generators $a^{\prime \prime} a^{\prime}$ or $a^{\prime} a^{\prime \prime}$ are inserted somewhere in $C_{z}$,

- new generators $a^{\prime \prime}$ and $a^{\prime}$ are introduced along with relations $a^{\prime} \sim a \triangleleft_{-} z$ and $a^{\prime \prime} \sim a^{\prime} \triangleright_{+} z$ or $a^{\prime} \sim a \triangleright_{-} z$ and $a^{\prime \prime} \sim a^{\prime} \triangleleft_{+} z$, and

- the outbound operand $a$ is replaced in its relation with $a^{\prime \prime}$ and the operator $a$ is replaced with $a^{\prime \prime}$ in the relations with inbound operands $x_{1}, \ldots, x_{i}$.

Conversely, a crossing-removing Reidemeister II move is available only when we have relations with opposite crossing signs $a^{\prime} \sim a \triangleleft_{-} z$ and $a^{\prime \prime} \sim a^{\prime} \triangleright_{+} z$ or $a^{\prime} \sim a \triangleright_{-} z$ and $a^{\prime \prime} \sim a^{\prime} \triangleleft_{+} z$, and order information including

$$
C_{a^{\prime \prime}} C_{a^{\prime}} C_{a}=\underline{x_{1} \ldots x_{i}} a^{\prime \prime}-a^{\prime} \underline{x_{i+1} \ldots x_{n}},
$$


and

$$
C_{z}=\ldots a^{\prime} a^{\prime \prime} \ldots z \text { or } C_{z}=\underline{\ldots a^{\prime \prime} a^{\prime} \ldots z} ;
$$

in this case we may delete the generators $a^{\prime \prime}, a^{\prime}$, their relations and replace any remaining instances of $a^{\prime \prime}$ with $a$.
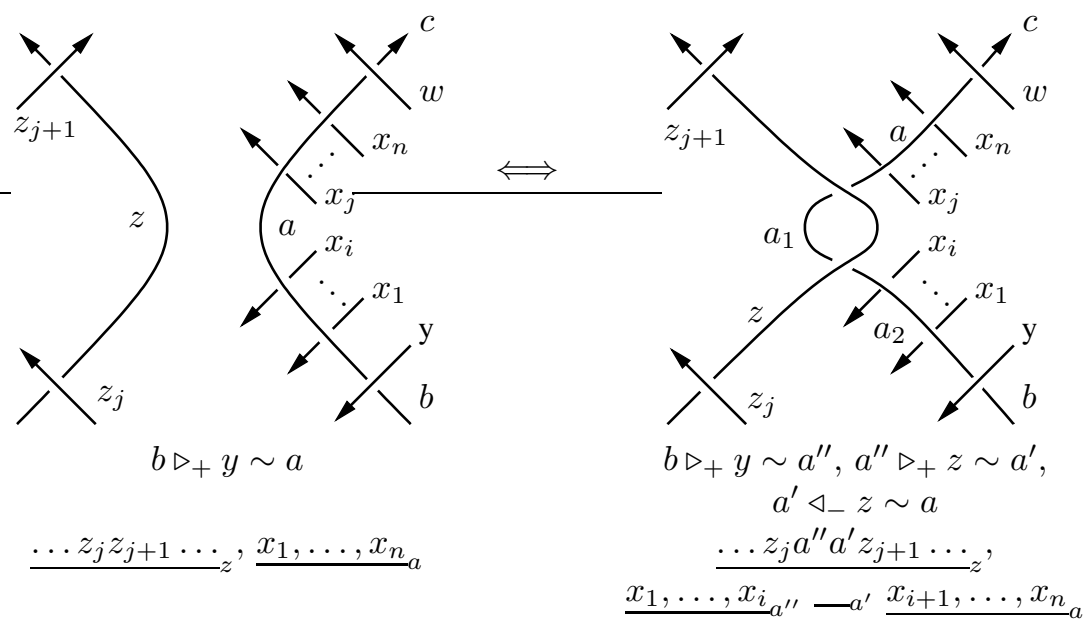

operator $a$ replaced with operator $a^{\prime \prime}$ in relations with inbound operand $x_{1}, \ldots, x_{i}$

Figure 7: Formal Reidemeister move II example

In the Reidemeister type III move, one generator is replaced with another, and since this generator does not appear as an operator in any relation, for simplicity we may use the same name for both the old and new generator. There are a number of cases, but in each case, we have a set of three short relations which get replaced by another set of three short relations with one relation the same, one (the defining relation for the generator which gets removed and re-added) changed, and the other relation changed by a Tietze move involving a rightdistribution.

The order information in a type III move changes by a cyclic permutation of the input operands around the central triangle formed by the three strands in the move. Since the cyclic order of the strands does not change in the move, the cyclic order of the arc-comparison classes also does not change. The arccomparison class corresponding to the top strand contains two of the pictured generators, the arc-comparison class of one end of the middle strand has one pictured generator either as its maximum or minimum element, and the arc- 
comparison class of the other end of the middle strand includes no pictured generators. The arc-comparison classes of the bottom strand generators are unaffected by the move.

The type III move fixes the cyclic order of the arc-comparison classes, and it changes the partial order of the generators inside the classes in a nice way. The two arc-comparison classes with operators on the middle strand are adjacent in the cyclic order, with the pictured generator at the end of one class next to the other. The other two generators with pictured terminal points are adjacent somewhere in the arc-comparison class of the top strand. The move changes both the positions occupied by the pictured generators and which generators fill those positions. The position occupied by the pair remains in place, while the position at the extreme end of one of the middle-strand classes moves to the other extreme of the adjacent class. The generators filling these positions then undergo a cyclic permutation.

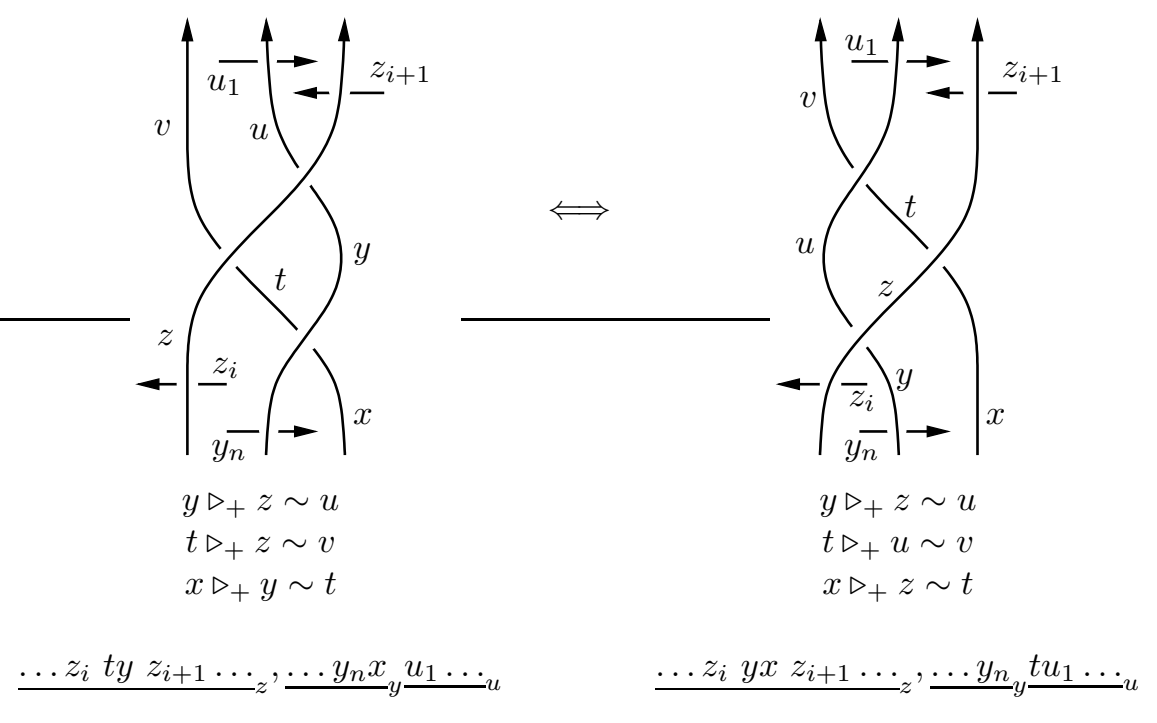

Figure 8: Formal Reidemeister move III example

The fact that two oriented virtual knots are, by definition, virtually isotopic iff they are related by Reidemeister moves implies the following:

Theorem 4.1 Two signed ordered knotlike quandle presentations present isotopic oriented virtual knots if and only if they are related by a finite sequence of formal Reidemeister moves. 
Note that in all three moves, the cyclic order of the arc-comparison classes is changed only by insertions of new generators and deletions of old ones. In particular, the cyclic order of the arc-comparison classes is an invariant of oriented virtual knot type, in the following sense:

Proposition 4.2 If $C_{x_{1}} \prec C_{x_{2}} \prec C_{x_{3}}$ in $Q=\langle X \mid R\rangle, Q^{\prime}=\left\langle X^{\prime} \mid R^{\prime}\right\rangle$ and $f: X \rightarrow X^{\prime}$ generates an isomorphism of quandles which is realizable by formal Reidemeister moves, then the cyclic order on $Q^{\prime}$ must include $C_{f\left(x_{1}\right)} \prec C_{f\left(x_{2}\right)} \prec$ $C_{f\left(x_{3}\right)}$.

While the cyclic order does not distinguish the two diagrams in figure 3 the full order information is different for these two. Specifically, the virtual knot diagram on the left has order information $\underline{a b}_{a \_}$, while the diagram on the right has order information $\underline{b a}_{a-b}$. Similarly, the order information for the nontrivial Kishino virtual knot on the left in figure 4 differs from that of the unknot on the right only by switching the order of the generators $a$ and $d$ in the arccomparison set $C_{a}$; the diagram on the left has order information $\underline{a d}_{a} \underline{c}_{b} \_c \underline{b}_{d}$, while the unknot diagram on the right has order information $\underline{d a}_{a} \underline{c}_{b} \underline{c}_{-} \underline{b}_{d}$.

However, it not the case that SOKQ presentations which differ only in the order information necessarily present non-isotopic virtual knots. For example, figure 9 shows two isotopic virtual knots constructed from SOKQ presentations which differ only in the order information. Moreover, a connected sum of the two virtual knots in this example with any other virtual knot will yield additional examples of possibly isotopic virtual knots with the same quandle but different order information. Indeed, compare figure 4 In particular, the order information itself is not an invariant of virtual knot type; rather, it is the equivalence class of order-information posets under the three Reidemeister moves which is an invariant of virtual isotopy.

The fact that these examples are non-classical, however, leaves open the question of whether there exist isotopic classical knots with SOKQ presentation differing only in the order information.

SOKQ presentations give a method for systematically listing all possible virtual knot diagrams with $n$ arcs. Namely, for a set of $n$ generators, choose ordered pairs of generators to be inbound and outbound operands. Then for each such pair, select an operator, quandle operation and coherent crossing sign, determining a knotlike quandle presentation. For each resulting signed knotlike quandle presentation, the operators divide the set of inbound generators into arc-comparison classes, and we then can list each possible ordering of the generators within the classes to complete an SOKQ presentation. For 


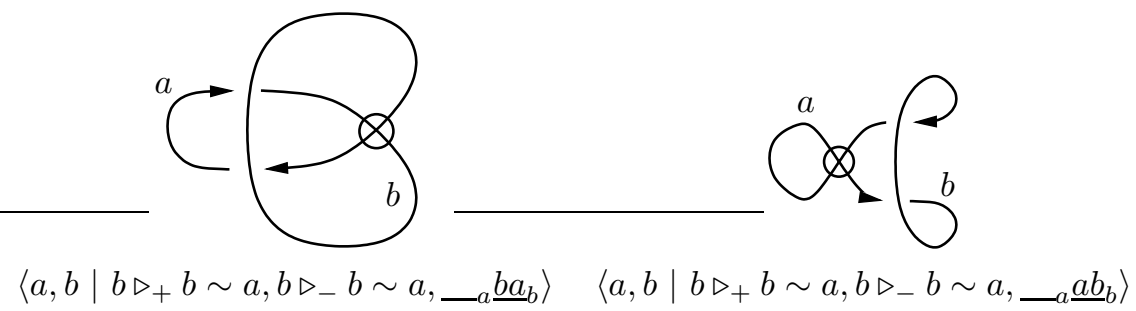

Figure 9: Two isotopic virtual knots whose SOKQ presentations differ only in the order information

example, the two diagrams in figure 3 are the only two possible diagrams, up to virtual moves, with the given knotlike quandle presentation. Note that the cyclic ordering of the classes is determined by the inbound/outbound pairing, though we can choose any ordering of the crossings within each arc class.

We can now ask what other invariants of virtual isotopy might be determined by the order information in a signed ordered knotlike quandle presentation; such an invariant is necessarily not determined by the quandle. The primary new invariant contributed by SOKQ presentations is the equivalence class of cyclically-ordered posets under the three formal Reidemeister moves. It is not yet clear to this author how to compare two such poset-classes, though the problem deserves further study.

Other possibilities include invariants derived from the poset-class. For example, we have the following:

Definition 4.1 Let $K$ be an oriented virtual knot diagram. Label the arcs in $K$ with the numbers $1,2, \ldots, n$ in the order in which they are encountered traveling around the knot in the direction of the orientation, so that the cyclic order of the arc comparison classes is $C_{1} \prec C_{2} \prec \ldots \prec C_{n}$. The endpoint ordering determines a permutation of $\{1,2, \ldots, n\}$, specifically an $n$-cycle, which we may regard as an element of the infinite cyclic group $\Sigma_{\infty}$. We then define the order-permutation group of the knot $K$ to be the subgroup of $\Sigma_{\infty}$ generated by these permutations for all diagrams of the oriented virtual knot $K$.

The full order information tells us which other permutations we must include in the generating set of the order-permutation group: a type I move inserts a number $i$ at some point along the arc $C_{i-1}$ and increments the arc labels $i, i+1, \ldots, n$ by 1 . This may be accomplished by composing the permutation corresponding to the pre-move diagram with the transposition $(x(n+1))$ where the insertion is after the symbol $x$, then composing with the cycle 
$(i(i+1) \ldots(n+1))$ to increment the arcs. A type II move is similar, inserting two adjacent numbers $i, i+1$ anywhere in the cycle and incrementing the arc labels $i, \ldots, n$ by 2 , done by composing the pre-move cycle with $(x(n+1)(n+2))$ or $(x(n+2)(n+1))$ then with $(i(i+1) \ldots(n+1)(n+2))$. Finally, type III moves simply compose the pre-diagram cycle with a 3 -cycle determined by the order information as described previously. While this group is infinitely generated, perhaps some more computable finite invariant may be derivable from it.

\section{$5 \quad$ Welded links}

We now turn to welded links. Looking at the forbidden move (figure 1) we observe that this move leaves invariant the cyclic order information but changes the ordering within the arc-comparison classes. Thus we would expect that welded links (ie welded isotopy classes of virtual link diagrams) should correspond to signed knotlike quandle presentations (ie without the order information). We shall see that this is indeed the case. Let us call a signed knotlike quandle presentation an $S K Q$ presentation for short.

We can define formal Reidemeister moves on SKQ presentations in an analogous way to those for SOKQ presentations. Indeed the definitions are given by simply omitting the order information from the preceding ones. We say that SKQ presentations are equivalent if they are related by a sequence of formal Reidemeister moves. A virtual link diagram gives an SKQ presentation as we saw earlier and furthermore Reidemeister moves, the detour move and the forbidden move $F_{h}$ on a diagram all correspond to formal Reidemeister moves. Thus we have a natural map $\Phi$ from oriented welded links to equivalence classes of SKQ presentations.

Theorem 5.1 The natural map $\Phi$ just described is a bijection.

Proof We can turn an SKQ presentation into an SOKQ presentation by choosing an arbitrary order for the arc-comparison classes, noting that the cyclic order of the classes is determined by the SKQ presentation, and hence by theorem 4.1 find a corresponding virtual link diagram. Furthermore a change in the chosen order can be realised as a product of adjacent interchanges each of which can be realised by an $F_{h}$ move. Thus we have a map from SKQ presentations to welded links. Now a formal Reidemeister move on an SKQ presentation corresponds to a formal Reidemeister move on corresponding SOKQ presentations and hence we have a map $\Psi$ from equivalence classes of SKQ presentations to welded links. It can readily be seen that $\Phi$ and $\Psi$ are inverse bijections. 


\section{The framed case}

We finish by observing that there is an analogous treatment for framed virtual links and framed welded links. Framed virtual links are equivalence classes under Reidemeister moves II and III of 4-valent graphs with vertices interpreted as crossings, or equivalently of framed virtual link diagrams under Reidemeister moves II and III and the detour move. For framed welded links the forbidden move $F_{h}$ is also allowed. It is worth commenting that for framed classical links it is necessary to include a double Reidemeister I move [6, page 370]. With the detour move available is it an easy exercise to check that this double move is unnecessary.

The corresponding algebraic object for framed links is the rack which has exactly the same definition as the quandle but with axiom (qi) omitted. We can define signed knotlike rack presentations and signed ordered knotlike rack presentations (SKR and SOKR presentations respectively) by copying the quandle definitions without change. Then completely analogous arguments show:

Theorem 6.1 The are bijections between the sets of isotopy classes of framed virtual links (respectively framed welded links) and the sets of equivalence classes of SOKR presentations (respectively SKR presentations) under the equivalence generated by formal Reidemeister moves II and III.

\section{Acknowledgments}

The author would like to thank Xiao-Song Lin and Jim Hoste, whose conversations the author found invaluable during the preparation of this paper, as well as Louis Kauffman and Scott Carter, whose comments at a conference started the author thinking about knot reconstruction from quandles. The author would also like to thank the referee and the editor, whose comments and corrections improved the paper considerably.

\section{References}

[1] J S Carter, D Jelsovsky, S Kamada, L Langford, M Saito, State-sum invariants of knotted curves and surfaces from quandle cohomology, Electron. Res. Announc. Amer. Math. Soc. 5 (1999) 146-156 MathReview

[2] J S Carter, S Kamada, M Saito, Stable equivalence of knots on surfaces and virtual knot cobordisms. J. Knot Theory Ramifications 11 (2002) 311-322 MathReview 
[3] H A Dye, Virtual knots undetected by 1 and 2-strand bracket polynomials, arXiv:math.GT/0402308

[4] R Fenn, M Jordan-Santana, L Kauffman, Biquandles and virtual links, Topology Appl. 145 (2004) 157-175 MathReview

[5] R Fenn, R Rimanyi, C Rourke, The braid-permtuation group, Topology 36 (1997) 123-135 MathReview

[6] R Fenn, C Rourke. Racks and links in codimension two, J. Knot Theory Ramifications 1 (1992) 343-406 MathReview

[7] R Fenn, C Rourke, B Sanderson, Trunks and classifying spaces, Appl. Categorical Struc. 3 (1995) 321-356 MathReview

[8] R Fenn, C Rourke, B Sanderson, The rack space, Trans. Amer. Math. Soc. (to appear) arXiv:math.GT/0304228

[9] M Goussarov, M Polyak, O Viro, Finite-type invariants of classical and virtual knots, Topology 39 (2000) 1045-1068 MathReview

[10] D Joyce, A classifying invariant of knots, the knot quandle. J. Pure Appl. Algebra 23 (1982) 37-65. MathReview

[11] L Kauffman, Virtual knot theory. European J. Combin. 20 (1999) 663-690. MathReview

[12] G Kuperberg, What is a Virtual Link? Algebr. Geom. Topol. 3 (2003) 587591 MathReview

University of California, Riverside

900 University Avenue, Riverside, CA 92521, USA

Email: knots@esotericka.org

Received: 14 September 2004 Revised: 29 March 2005 